\title{
Long-term use of the wearable cardioverter-defibrillator: prolonging life or prolonging indecision?
}

\author{
Evan Adelstein ${ }^{1}$ (1)
}

Received: 7 November 2016 / Accepted: 16 November 2016/Published online: 26 November 2016

(C) Springer Science+Business Media New York 2016

Predicting future risk is difficult. Predicting sudden cardiac death (SCD) is a particular challenge, given its finality, suddenness, inherent unpredictability, and medicolegal ramifications. Yet the task of prognosticating SCD risk is often thrust upon electrophysiologists in the setting of a new cardiomyopathy.

The implantable cardioverter-defibrillator (ICD) mitigates SCD risk in patients at increased risk for ventricular arrhythmias. Its effectiveness is largely not questioned [1], although the appropriate population who should receive an ICD is still being refined [2]. The wearable cardioverter-defibrillator (WCD) was approved by the U.S. Food and Drug Administration in 2001 as a temporary device that continuously monitors cardiac rhythm and is highly effective in terminating potentially lethal arrhythmias [3-5]. It has been marketed for use in patients deemed at elevated risk of SCD from ventricular arrhythmias in whom a permanent ICD is either contraindicated or under consideration. To date, there have been several publications describing its use in clinical registries [5, 6], national experiences $[4,7]$, and single-center studies [8], primarily as a short-term means of mitigating risk while patients undergo more comprehensive evaluation or titration of medical therapy. There are, at present, no currently

Editorial response to National experience with long-term use of the wearable cardioverter-defibrillator in patients with cardiomyopathy

I am the sole author of this editorial.

Evan Adelstein

adelec@upmc.edu

1 University of Pittsburgh, 200 Lothrop Street, PUH B535, Pittsburgh, PA 15213, USA published randomized WCD clinical trials demonstrating a mortality benefit.

In this issue of the Journal of Interventional Cardiac Electrophysiology, Lamichhane et al. [9] describe the U.S. experience with long-term WCD use in patients with ischemic or non-ischemic cardiomyopathy, primary or secondary prevention indication, no prior ICD, and LVEF $\leq 35 \%$. Remarkably, 220 patients were prescribed with the WCD for $\geq 1$ year, and the median number of actual use days was 394 . Despite such prolonged use, over half of all the patients wore the WCD $>80 \%$ of the day.

Long-term WCD use has not been heretofore described in the literature. In the present study, some reasons cited for longterm use rather than ICD implantation included continued optimization of medical therapy (7.7\%), non-compliance for follow-up (10\%), patient postponement or unwillingness to have an ICD (16.8\%), ongoing evaluation for an ICD (34.5\%), and contraindication to an ICD because of cancer, poor prognosis, or vascular access issues $(23.2 \%)$. The incidence of appropriate shocks was $4.1 \%$; 9 of the 220 patients received a shock, 3 of whom already had prior sustained ventricular arrhythmias (i.e., secondary prevention indication). Of the remaining $6(2.9 \%)$ primary prevention patients, 4 had ischemic cardiomyopathy and 2 had non-ischemic cardiomyopathy. One non-ischemic patient had reported WolffParkinson-White syndrome, and another had chronic lymphocytic leukemia.

The WCD has proven effectiveness in terminating tachyarrhythmias. In this study, the WCD successfully terminated $92 \%$ of VT/VF episodes. However, there is no mention of cost, either personal or monetary. On a psychosocial level, wearing the WCD consistently for a year requires tremendous personal fortitude. If one assumes a monthly fee of $\$ 3000$ for the WCD [8], its cost over 1 year is similar to an ICD, yet after that year, 
additional protection against $\mathrm{SCD}$ will require additional financial input.

The authors should be applauded for this novel manuscript, yet the WCD was never intended for long-term use. The most disconcerting aspects of this manuscript are the unorthodox reasons listed for prolonged WCD use. Appropriate WCD shocks are relatively rare during the period of medical titration [8], particularly among non-ischemic primary prevention patients, so long-term WCD use in this population may reflect physicians' perceived risk rather than actual risk. Immediately after myocardial infarction, ICDs have no demonstrated survival benefit; therefore, the role of the WCD in this population is also unclear $[10,11]$. Non-compliance should raise the question as to whether a patient should receive any defibrillator, either wearable or implantable, not necessarily prompt protracted WCD use. Another unsettling aspect of the manuscript is the number of patients prescribed a long-term WCD in the context of contraindications to an ICD because of comorbid medical conditions. SCD may be preferable to a protracted, potentially uncomfortable death from other causes, such as heart failure or cancer. Were these often difficult concepts discussed with patients, or did physicians focus too much on quantity rather than quality of life? Or was the WCD thrust upon them without discussion of its ramifications, responsibilities, and pitfalls? Many patients who receive an ICD, for example, receive inadequate counseling [12], but little is known about whether similar circumstances surround WCD discussions. The WCD is not meant to obviate the need to make a decision to implant or not implant an ICD, yet it is in this capacity that the WCD seemingly was used in the majority of the patients included herein.

How should this study be incorporated into clinical practice? For now, this single study should not change current indications for WCD use, which should be limited in duration and to patients at the highest risk for SCD while awaiting ICD implantation or additional diagnostic testing. Of greater concern are the implications of this study regarding physicians' attitudes towards prognosticating SCD risk. Hopefully, the WCD is not becoming a crutch that allows physicians to dodge difficult decisions and conversations with patients while avoiding any medicolegal culpability. The implications of wearing the WCD for months on end should not be minimized; aside from physical inconvenience, the device serves as a constant reminder to the patient of his/her risk of SCD, and the WCD may heighten perceived risk when compared to actual risk in primary prevention patients. Inappropriate shocks also remain a concern; while inappropriate shocks are by no means limited only to the WCD, in the present study, they were equally prevalent as sustained ventricular arrhythmias requiring a shock [9].
This study unfortunately does not provide randomized data regarding the WCD, and in describing long-term WCD use, it now raises the question: does the WCD save lives or save physicians from making decisions? We need a prospective clinical trial to provide randomized data, and we need a health care delivery system that places value not just on performing interventions but also on discussing the rationale for non-intervention.

\section{Compliance with ethical standards}

Conflict of interest The author declares that he has no conflict of interest.

\section{References}

1. Hohnloser SH, Israel CW. Current evidence base for use of the implantable cardioverter-defibrillator. Circulation. 2013;128:17283.

2. Tung R, Zimetbaum P, Josephson ME. A critical appraisal of implantable cardioverter-defibrillator therapy for the prevention of sudden cardiac death. J Am Coll Cardiol. 2008;52:1111-21.

3. Reek S, Geller JC, Meltendorf U, et al. Clinical efficacy of a wearable defibrillator in acutely terminating episodes of ventricular fibrillation using biphasic shocks. Pacing Clin Electrophysiol. 2003;26:2016-22.

4. Klein HU, Meltendorf U, Reek S, et al. Bridging a temporary high risk of sudden arrhythmic death. Experience with the wearable cardioverter defibrillator (WCD). Pacing Clin Electrophysiol. 2010;33:353-67.

5. Feldman AM, Klein H, Tchou P, et al. Use of a wearable defibrillator in terminating tachyarrhythmias in patients at high risk for sudden death: results of the WEARIT/BIROAD. Pacing Clin Electrophysiol. 2004;27:4-9.

6. Kutyifa V, Moss AJ, Klein H, et al. Use of the wearable cardioverter defibrillator in high-risk cardiac patients: data from the prospective registry of patients using the wearable cardioverter defibrillator (WEARIT-II registry). Circulation. 2015;132:1613-9.

7. Chung MK, Szymkiewicz SJ, Shao M, et al. Aggregate national experience with the wearable cardioverter-defibrillator: event rates, compliance, and survival. J Am Coll Cardiol. 2010;56:194-203.

8. Singh M, Wang NC, Jain S, et al. Utility of the wearable cardioverter-defibrillator in patients with newly diagnosed cardiomyopathy: a decade-long single-center experience. J Am Coll Cardiol. 2015;66:2607-13.

9. Lamichhane M, Gardiner JC, Bianco NR, et al. National experience with long-term use of the wearable cardioverter-defibrillator in patient with cardiomyopathy. J Interv Card Electrophysiol 2016. doi:10.1007/s10840-016-0194-6

10. Hohnloser SH, Kuck KH, Dorian P, et al. Prophylactic use of an implantable cardioverter-defibrillator after acute myocardial infarction. N Engl J Med. 2004;351:2481-8.

11. Steinbeck G, Andresen D, Seidl K, et al. Defibrillator implantation early after myocardial infarction. N Engl J Med. 2009;361:142736.

12. Hauptman PJ, Chibnall JT, Guild C, et al. Patient perceptions, physician communication, and the implantable cardioverter-defibrillator. JAMA Intern Med. 2013;173:571-7. 